\title{
A review of cricket fielding requirements
}

\author{
${ }^{1}$ Sport Performance Research Institute, AUT University, Auckland, New Zealand \\ ${ }^{2}$ New Zealand Cricket, Christchurch, New Zealand \\ ${ }^{3}$ School of Exercise and Health Sciences, Edith Cowan University, Perth, Australia \\ ${ }^{4}$ Nelson Mandela Metropolitan University, Port Elizabeth, South Africa
}

D MacDonald, ${ }^{1,2}$ MSc; J Cronin, ${ }^{1,2,3} \mathrm{PhD} ; \mathrm{J}$ Mills, ${ }^{2}$ Grad Dip; M McGuigan, ${ }^{1,3} \mathrm{PhD}$; R Stretch, ${ }^{4}$ Phil

Corresponding author: D MacDonald (dani.macdonald@aut.ac.nz)

Cricket is played in three formats at elite level: Test, One Day and Twenty20. Fielding is an important component of cricket, as all players are obliged to field. However, there is a paucity of literature on fielding compared with that on batting and bowling. We review the available literature in terms of technical, mental, physiological and physical factors important to fielding, to identify knowledge gaps and better understand the performance requirements of fielding in cricket.

S Afr J SM 2013;25(3):87-92. DOI:10.7196/SAJSM.473

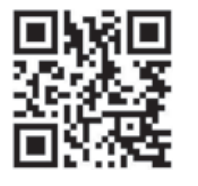

Internationally, three formats of cricket are played at the elite level: Test, One Day and Twenty20. ${ }^{[1]}$ All players bat and field, while only some players bowl and one person keeps wicket. Dismissing a batsman can be achieved in different ways, some specific to fielders; hence, catching and throwing are vital skills. Common requirements for these skills are speed and accuracy. ${ }^{[2]}$ As well as dismissing batsmen, the role of fielders includes saving runs, particularly in the shorter formats of the game. Therefore, optimising the movements and skills required to successfully field can have an important influence on the game. However, despite the adage that 'catches win matches', research into fielding is sparse compared with that into batting and bowling. ${ }^{[3]}$ The purpose of this review was therefore to investigate and critique the existing knowledge of fielding in cricket, with the intent of better understanding the performance demands of fielding.

Methods

For the purpose of this review, fielding performance was divided into a number of components (Fig. 1) which were systematically reviewed. Reviewed literature included peerreviewed articles and book chapters. The requirements of the wicket-keeper are not discussed here, as the demands of this position have been reviewed previously. ${ }^{[4]}$
The literature search was conducted using search engines (PubMed, SportsDiscus and ScienceDirect). The search terms 'fielding', 'wicket-keeping', 'catching', 'cricket' and other related terms were used in various combinations to search for articles. The reference lists of articles found were assessed to extend the search. As fielding was the focus of this review, articles specifically focusing on the wicket-keeper were excluded from the review. Additionally, cricket-relevant chapters from edited books were included.

\section{Technical}

In cricket, the playing field is not of fixed dimensions. According to the laws of the game, 'the playing area shall be a minimum of 150 yards $(137.16 \mathrm{~m})$ from boundary to boundary square of the pitch, with the shorter of the two square boundaries being a minimum of 70 yards $(64.01 \mathrm{~m}){ }^{\prime} \cdot{ }^{[5]}$ Due to the large and varying size of the playing field, the skills of fielding in cricket will vary considerably depending on where fielders are placed. Here, the fielding positions have been categorised as close (e.g. slips and short leg), inner-circle and outer-circle (Fig. 2).

Shilbury ${ }^{[6]}$ researched the frequency of fielding skills for 25 defined positions, and the fielding patterns of individual players of an 'A'-grade cricket team playing first-class multi-day cricket. The data were divided into four skill categories: fielded ball, fielded ball and throw, fielded ball and under-arm return, and catches and attempted catches. The author reported the frequency and skills required in 25 fielding positions. The positions which featured the most were cover (12\%), mid-off (10\%) and mid-on (9\%), respectively.

Cover has traditionally been considered a position that requires good attacking skills, such as being able to move towards the ball, field and throw quickly, often from unbalanced positions. ${ }^{[6]}$ However, only $13 \%$ of cover's fielding contacts required attacking skills; the majority of actions were defensive

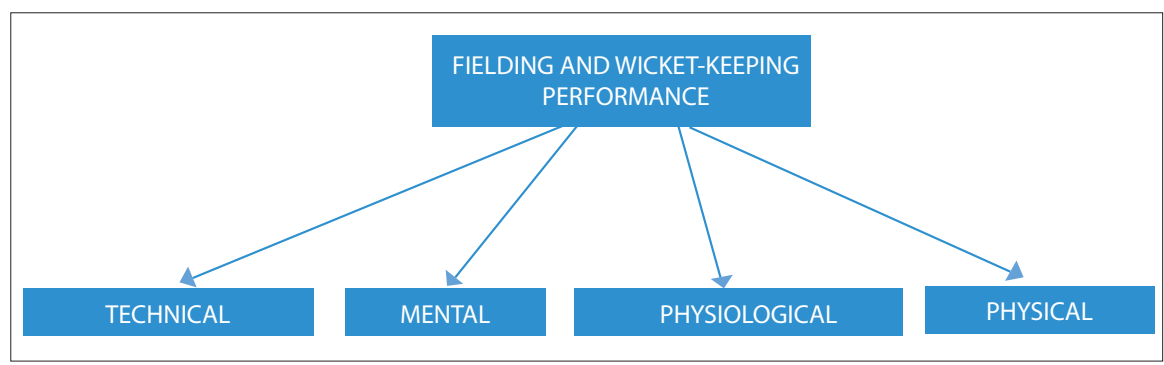

Fig. 1. Aspects of fielding performance. 


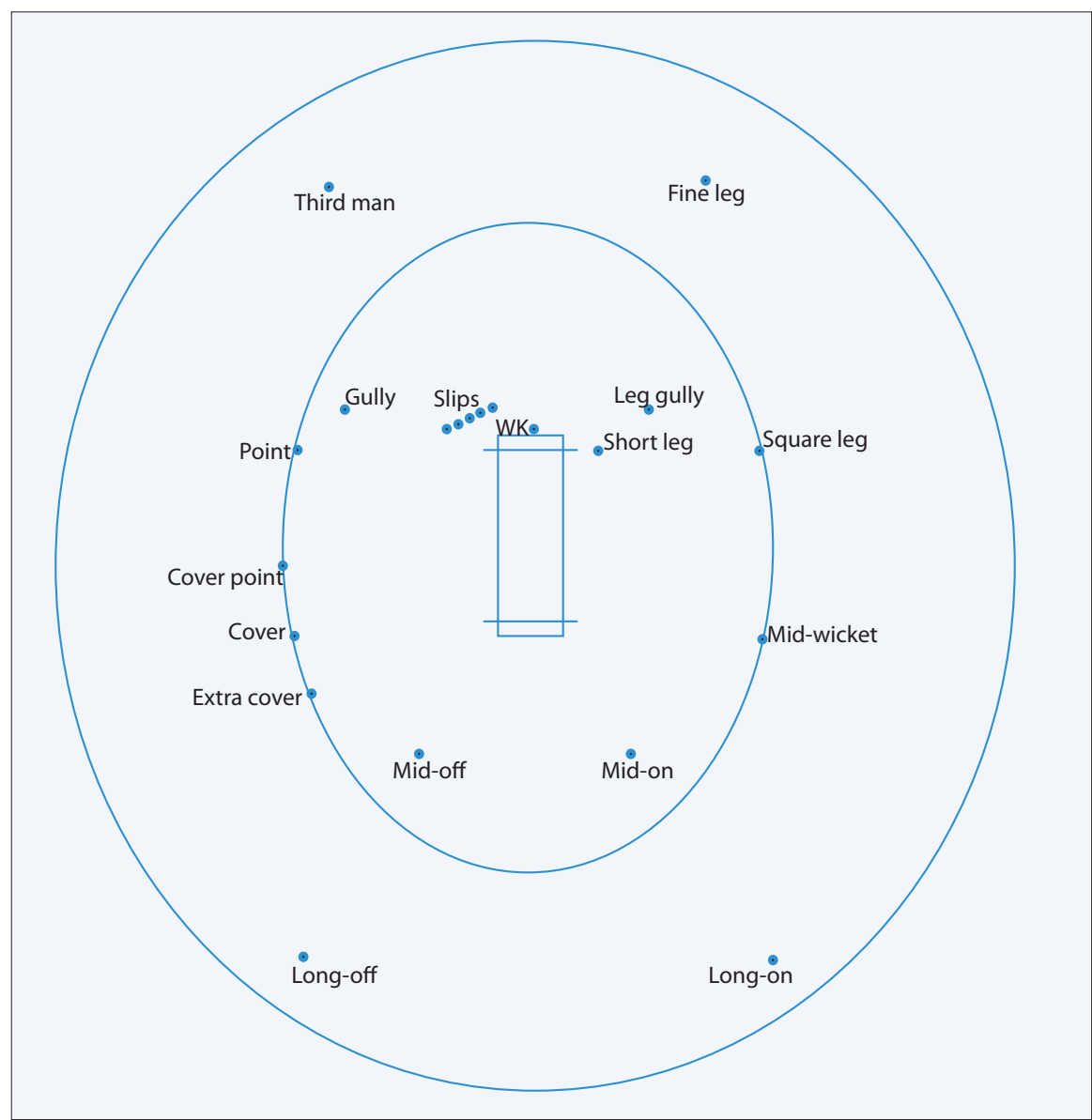

Fig. 2. Pitch map showing the different fielding categories. $(W K=$ wicket keeper. $)$

and required practically no diving or lateral movements. This finding is not consistent with conventional wisdom.

Shilbury's study is dated (1990), and included data from only six domestic games. Given the developments in the game since the 1990s, research based on a larger number of international matches would be more appropriate and useful. These findings will assist the development of assessment and training protocols for the different formats of the game at the highest level.

In One Day cricket, matches began to be played into the night, and the ball colour was changed from red to white to be seen better under floodlights. Scott et al. ${ }^{[7]}$ investigated the effect of light levels and ball colour on catching, particularly for slip fielders in simulated field conditions. Photoelectric timing gates were placed in front of a ball projection machine and lever micro-switches were placed on the thighs of the players to establish reaction times to balls projected at a speed of $20 \mathrm{~m} / \mathrm{s}$ over a distance of $8.4 \mathrm{~m}$. The speed was considered representative of the demands of slip-catching performance, and was the upper limit of speeds safe for use under laboratory conditions. Catching performance was scored using a scale adapted from Wickstrom, ${ }^{[8]}$ which rated catches from 0 (no ball contact at all) to 5 (clean catch). analysis of variance (ANOVA), no significant effects were noted for catching performance and movement initiation times for ball or light levels. The authors concluded that the change in ball colour or diminishing light levels were not detrimental to performance. However, the sample size was very small $(n=5)$, which might have accounted for the lack of statistical significance. Also, the testing protocol required players to assume a standing position that may not be normal under game conditions.

The effect of a visual-perceptual training programme on fielding in cricket has been investigated using a test-retest design involving a 6-week training intervention. ${ }^{[9]}$ The tests involved an in situ fielding test, and athletes were required to react (predict and After performing a 2 (ball) $\times 3$ (light level) move in the direction that the ball was hit) to a life-sized video projection of a batsman hitting strokes, with the video occluded at the point of ball contact. The video was filmed from the perspective of 3 different fielding positions: extra cover, mid on and mid off. Moving in the correct direction for each video assessed decision accuracy.

The training group underwent an additional 3 perceptual training sessions per week in addition to the on-field training programme undertaken by the control group. The training group performed significantly better than the control group in the two tests. It was concluded that while 6 weeks of regular onfield training may lead to improvements in fielding performance, greater advantages could be gained when this is combined with visual perceptual training sessions.

A fielder's ability to throw a ball over considerable distance with speed and accuracy, if aiming for a run out, requires excellent throwing technique. ${ }^{[10]}$ The requirements are specific for the different field positions. The slips mostly intercept a fast moving ball coming off the edge of the bat and reaching them below chest height. ${ }^{[1]]}$ They have little need for throwing long distances. In-fielders require good reactive ability to catch a ball falling from above their heads and strong overarm throwing ability ${ }^{[3]}$ to attempt run-outs. Outfielders often have to cover a considerable distance, so sprinting ability is vital, and they need to throw accurately over long distances. ${ }^{[6]}$ Good techniques are not only essential to win matches; they also minimise the risk of injury. ${ }^{[2]}$

Synchronised high-speed video cameras ${ }^{[1]}$ have been used to study the biomechanics of throwing. Distinguishing different throwing techniques has led to the identification of important performance variables. ${ }^{[1]}$ The relationship between over-arm throwing velocity and accuracy in elite and sub-elite cricketers was investigated using a specifically designed throwing test. ${ }^{[8]}$ A speed-accuracy trade-off was detected. Subjects improved accuracy scores at velocities of $75-85 \%$ of maximal throwing velocity. Senior elite players performed better than other groups. No research into side- or underarm throwing in cricket has been found. Using the correct technique is crucial for success; the lack of empirical data in this area limits the development of optimal training programmes. 


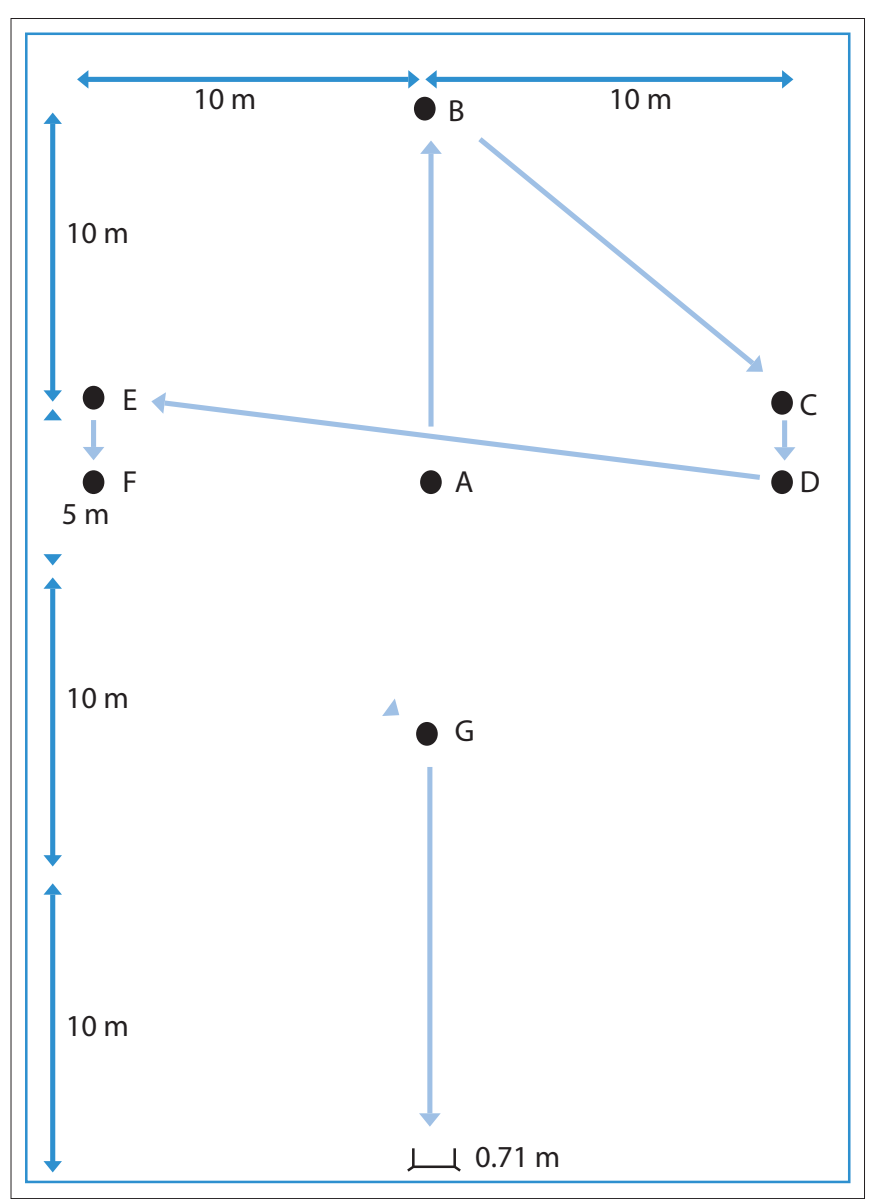

Fig. 3. Schematic of the fielding test used by Stretch and Goslin. ${ }^{[2]}$

For skills development, player selection and talent identification, it is important to test correctly for skill and movement efficacy. This can assist a coach to detect strengths and weaknesses in performance and to identify the specific training needs of the individual ${ }^{[12]}$ Stretch and Goslin $^{[12]}$ devised a set of cricket skills tests, encompassing all components of the game. With regards to fielding, the majority of runouts occur between $10 \mathrm{~m}$ and $35 \mathrm{~m},{ }^{[13]}$ and these were the distances tested in the fielding test (Fig. 3).

At point $\mathrm{A}$, the fielder was required to catch a thrown ball, over-arm throw at the target and then move as quickly as possible to point $B$. At points $\mathrm{B}, \mathrm{C}, \mathrm{D}, \mathrm{E}$, and $\mathrm{F}$, the fielder was required to pick a ball up from the ground and over-arm throw at the target; point $\mathrm{G}$ required a pick up and under-arm throw at the target, followed by a final sprint to the target. The timer started when the fielder touched the first ball and ended when he had run through the target. A time penalty ( $3 \mathrm{~s}$ ) was given if he dropped the balls or a throw did not go through the target at any time during the test.

The authors and coaches also used their knowledge of the game to determine, subjectively, the players' potential success in a match. ${ }^{[12]}$ The validity of the fielding tests was tested by comparing the objective tests to the subjective opinion of coaches. The relationship between the objective fielding test scores and the subjective fielding evaluation was low $(r=0.47 ; p<0.05)$; however, the sample size was relatively large $(n=155)$, hence the authors decided that the lower correlation was acceptable. The diagnostic utility of this test could be questioned, given the composite nature of the test i.e. many skills assessed within one test.

\section{Mental}

Cricket requires inordinate physical skill and mental aptitude, including the ability to concentrate intensely for very long periods, for which a high level of physical fitness cannot fully compensate. ${ }^{[14]}$ Fielders have to concentrate on every ball of the innings, regardless of their positions. They have to be able to maintain concentration for the entire duration of an innings (ranging from approximately 90 minutes in a T20 innings to a total of 6 hours per day in a Test match), through changing conditions as play progresses through the day. However, studies on the mental aspect of cricket have focused on batting only; $;^{[15-17]}$ no research, to the authors' knowledge, has addressed the mental aspects of fielding.

\section{Physiological}

The most prevalent approach to quantifying the physiological demands of cricket is time-motion analysis using global positioning satellite (GPS) units. Rudkin and O'Donoghue ${ }^{[18]}$ performed 27 observations of a fielder positioned at cover point, during first-class multi-day games. They used the CAPTAIN time-motion analysis system to define seven movement classes: stationary, walking, shuffling (rapid non-running movement of the feet), jogging, running, low-intensity fielding and high-intensity fielding. It was found that the cover point fielder spends the majority $(94.2 \%$; standard deviation $(\mathrm{SD}) \pm 2.4)$ of match time in stationary activity and walking, while high-intensity activities represented just $1.6 \%$ ( $\mathrm{SD} \pm 0.8$ ) of movement activity. It was concluded that first-class fielding entails less high-intensity exercise than other team sports such as hockey and soccer. However, the conclusions are of limited value, as only one fielding position was analysed.

Time-motion studies could help develop knowledge of positional differences in workload between the different formats of cricket, allowing conditioning coaches to prescribe game-specific training programmes. ${ }^{[1]}$ Petersen et al.$^{[1,19,20]}$ have conducted several studies using GPS technology, investigating physiological demands of performance in the three different formats of the game. Unlike in the study by Rudkin and O'Donoghue, ${ }^{[18]}$ five movement categories were established (standing/walking, jogging, running, striding and sprinting) and the three different cricket formats were investigated.

Table 1 summarises time-motion analyses for each format of cricket. In summary, it seems that fielding intensity is greatest in a T20 match and fielders covered approximately the same distances in One Day and Test cricket.

Petersen et al. ${ }^{[19]}$ tested the validity and reliability of three commercially available sports GPS units to monitor cricket-specific movement patterns. They found disparate and inconsistent measures for the validity and reliability of low- and high-intensity activities. They advised that conditioning coaches should be aware of the likely under-reporting of high-intensity activity and over-reporting of lowintensity efforts when using GPSs in training. All studies detailed thus far fail to document the physiological demands of the different fielding positions because, generally, fielders - without distinction are compared with bowlers and batsmen.

\section{Physical}

The physical aspects of performance have been investigated with respect to injury incidence and prevention, particularly for fast bowling and throwing, but little investigation into the physical aspects of fielding 
Table 1. Summary of findings from time-motion studies by Petersen et al. ${ }^{[19,20]}$

\begin{tabular}{|c|c|c|c|}
\hline \multirow[b]{3}{*}{ Variable $^{\star}$} & \multicolumn{3}{|c|}{ Study } \\
\hline & \multirow{2}{*}{$\begin{array}{l}\text { Quantifying positional } \\
\text { movement patterns in } \\
\text { Twenty } 20 \text { cricket }^{[19]}\end{array}$} & \multicolumn{2}{|c|}{$\begin{array}{c}\text { Comparison of player movement patterns between } \\
\text { ODI and Test cricket }{ }^{[20]}\end{array}$} \\
\hline & & ODI & Test \\
\hline Position & Fielders $(n=14)$ & Fielders $(n=17)$ & Fielders $(n=25)$ \\
\hline \multicolumn{4}{|l|}{ Distance per hour (m) } \\
\hline Walking $(0-2.0 \mathrm{~m} / \mathrm{s})$ & $3286( \pm 726)$ & $2419( \pm 708)$ & $2263( \pm 629)$ \\
\hline Jogging $(2.0-3.5 \mathrm{~m} / \mathrm{s})$ & $1532( \pm 361)$ & $616( \pm 272)$ & $621( \pm 135)$ \\
\hline Running $(3.5-4.0 \mathrm{~m} / \mathrm{s})$ & $377( \pm 156)$ & $147( \pm 62)$ & $137( \pm 44)$ \\
\hline Striding $(4.0-5.0 \mathrm{~m} / \mathrm{s})$ & $497( \pm 316)$ & $159( \pm 89)$ & $166( \pm 62)$ \\
\hline Sprinting $(\geq 5 \mathrm{~m} / \mathrm{s})$ & $416( \pm 265)$ & $90( \pm 73)$ & $155( \pm 71)$ \\
\hline Total distance $(\mathrm{m})$ & $6106( \pm 981)$ & $3430( \pm 883)$ & $3342( \pm 759)$ \\
\hline \multicolumn{4}{|l|}{ Time (s) } \\
\hline Walking and jogging & $3263( \pm 187)$ & $3504( \pm 46)$ & $3496( \pm 30)$ \\
\hline Running, striding and sprinting & $275( \pm 146)$ & $91( \pm 45)$ & $104( \pm 30)$ \\
\hline \multicolumn{4}{|l|}{ Sprint } \\
\hline Number & $23( \pm 14)$ & $6( \pm 4)$ & $8( \pm 4)$ \\
\hline Mean sprint distance $(\mathrm{m})$ & $17( \pm 4)$ & $15( \pm 4)$ & $18( \pm 5)$ \\
\hline Maximum sprint distance (m) & $54( \pm 23)$ & $34( \pm 12)$ & $43( \pm 15)$ \\
\hline Maximum sprinting speed $(\mathrm{m} / \mathrm{s})$ & $8.6( \pm 1.1)$ & $7.9( \pm 1.2)$ & $8.5( \pm 0.9)$ \\
\hline \multicolumn{4}{|l|}{ High-intensity efforts } \\
\hline Number & $98( \pm 43)$ & $34( \pm 17)$ & $34( \pm 11)$ \\
\hline Effort duration & $2.8( \pm 0.4)$ & $2.6( \pm 0.3)$ & $3.1( \pm 0.3)$ \\
\hline Recovery between (s) & $45( \pm 21)$ & $134( \pm 73)$ & $116( \pm 37)$ \\
\hline \multicolumn{4}{|l|}{$\mathrm{ODI}=$ One Day International; $\mathrm{SD}=$ standard deviation. } \\
\hline * Values are expressed as mean $( \pm S D)$ & & & \\
\hline
\end{tabular}

has taken place. In this section, physical aspects such as anthropometry, strength, speed and aerobic and anaerobic fitness will be considered.

\section{Anthropometry}

Several studies have investigated the anthropometric profile of firstclass cricketers, ${ }^{[21-25]}$ mostly by comparing groups of players, such as batsmen, bowlers or all-rounders. The measures used mass and stature measurement and the sum of seven skin folds (biceps, triceps, subscapular, supra-iliac, abdominal, thigh and medial calf). Portus et al. ${ }^{[25]}$ extensively researched the characteristics of Australian players. However, these findings are probably not valid globally given ethnic differences in stature. While other physical attributes would be considered when deciding where to place players in the field, certain anthropometric characteristics may make players suited to specific positions. Nevertheless, the anthropometry for specific fielding positions has not been explored.

\section{Aerobic and anaerobic fitness}

With modern cricket, players can be expected to tour for up to eleven months of the year; therefore, physical fitness is increasingly important. The only study ${ }^{[26]}$ that focused specifically on the calorific energy demand of cricketers was performed in 1955, and its validity in representing the demands of modern players would seem problematic.

More recent research has indicated that cricketers generally rely on aerobic energy supply and that the rates of energy expenditure of cricket are relatively low; with the exception to this being fast bowlers during a bowling spell ${ }^{[21,26]}$ and fielders sprinting after the ball. This generalisation is supported by the findings of time-motion analyses. ${ }^{[1,19,27]}$

The multi-stage fitness test is recommended to test aerobic power as it is inexpensive, easy to administer and applicable to many team sports with respect to the stop, start and change-of-direction movement patterns. ${ }^{[24]}$ One study ${ }^{[14]}$ showed that cricketers had a higher shuttle run number when compared with rugby union players, with a $\mathrm{VO}_{2 \max }$ of $\sim 60 \mathrm{ml} / \mathrm{kg} / \mathrm{min}$. Johnstone and Ford ${ }^{[21]}$ established physical fitness profiles of cricketers grouped into bowlers and batsmen $(n=15)$ using this test. The authors recorded the number of completed shuttles (12.4; SD \pm 0.9 ), end heart rate (190.4 bpm; $\mathrm{SD} \pm 11.2)$ and predicted the $\mathrm{VO}_{2 \max }(54.9 \pm 3.7)$. The researchers 
concluded that the $\mathrm{VO}_{2 \max }$ results of cricketers were superior to that of the general population. ${ }^{[21]}$ These results are comparable with the normative data presented for cricket players in Physiological Tests for Elite Athletes. ${ }^{[25]}$

Anaerobic fitness has generally been tested using repeated sprint tests. Johnstone and Ford ${ }^{[21]}$ found that the running speed for each of the groups was similar, although the bowlers achieved moderately better results $(1.5 \%)$ in maximal repeated sprint tests than the batsmen. Sprint tests in cricket have typically varied in distance (10 - $40 \mathrm{~m}$ ); therefore, it is difficult to compare results between studies. Johnstone and Ford ${ }^{[21]}$ recommended that the future assessment of cricket-specific speed should use short distances of $5-15 \mathrm{~m}$, because these may be associated with higher levels of match-winning fielding performance. However, sprint testing over a large range of distances may be justified because the size of the pitch that players will have to cover varies in distance depending on fielding position.

In summary, cricket players require a high level of aerobic fitness in order to play for up to 6 hours per day, with intermittent, short bursts of high-intensity effort that requires contribution from the anaerobic energy system. Whether these demands differ as a function of fielding position has not been researched. It may be that a position such as slips with potentially less aerobic demands may benefit from greater doses of high-intensity reactive training. Nevertheless, given the length of the international cricket season and the tour demands of cricketers, superior aerobic fitness will assist players in recovery and sustaining performance at the highest level.

\section{Strength, power and speed \\ Upper body}

The results of studies on strength and power profiles of cricketers have, thus far, been ambiguous and seem to lack logical or face validity. Johnstone and Ford, ${ }^{[21]}$ for example, measured upper-body strength and power using a medicine ball throw and timed press-up tests. There were marked differences between batsmen and bowlers; the batsmen were superior in the timed press-up tests, but the bowlers produced greater backward throws. However, the significance of these results and their relation to performance is unclear. There is no research on specific fielding positional demands, nor have normative data for each fielding position been established. Nevertheless, it appears that different strength requirements may be needed for different fielding positions (e.g. the throwing demands of an outfielder v. a slip fielder).

\section{Lower body}

Leg strength and power are important for cricket fielders as they contribute to the speed and agility required for fielding. However, there has been little research on the lower-body strength profile of cricketers. Johnstone and Ford ${ }^{[21]}$ tested lower-body strength and explosive power using a counter-movement jump and repeated vertical jump test. The authors suggested that tests such as the counter-movement jump give an indication of slow stretchshortening cycle performance, and found that there were negligible differences between bowlers and batsmen. Bourdon et al. ${ }^{[24]}$ also recommended a series of tests for profiling the physical fitness of elite cricketers; the lower-body tests included a vertical double-leg jump, abdominal strength stage test, straight sprint speed $(10,20$ and $40 \mathrm{~m}$ ) and a run 3 agility test. While the abdominal stage test is lauded as particularly important for fast bowlers, the authors noted that batsmen and fielders would benefit from good abdominal strength during long periods in the field or at the batting crease.

The leg-power demands of fielding are little understood; however, a study comparing cricketers with rugby players found no significant differences in leg press, bench press and $35 \mathrm{~m}$ sprints. ${ }^{[14]}$ For example, there is little logic in using only a vertical jump test when research and observation show that fielders need to move in all directions and consequently need multi-directional lower-leg strength and power. Correctly assessing the multi-planar movement ability of fielders should lead to better training programmes.

\section{Conclusion}

There is a paucity of scientific information on the performance demands of fielding across all the areas of interest discussed in this review article, i.e. technical, physical, etc. There seems to be little appreciation of the technical requirements related to different fielding positions. From the scant literature available, it is possible to deduce that cricket fielding is, in general, a low-intensity activity that requires intermittent bursts of explosive movement. However, conventional wisdom on several aspects of the game is not supported by scientific evidence. Given that fielding is an essential component to winning matches, the lack of research in this area is disconcerting. A systematic research programme covering all components of all game formats would be beneficial.

Existing knowledge could be complemented by obtaining the considered opinions and insights of coaches and players and by carrying out detailed video and notational analyses. The results would provide greater insight into the skill and movement requirements associated with the different field positions. This information would also provide a framework for the design of fielding-specific assessments, which should enable the development of more focused training, conditioning and coaching protocols. This should enhance fielding performance and contribute to the ultimate goal of winning matches.

\section{References}

1. Petersen C, Pyne D, Dawson B, Portus M, Kellett A. Movement patterns in cricket vary by both position and game format. J Sports Sci 2010;28(1):45-52. [http://dx.doi. org/10.1080/02640410903348665]

2. Freeston J, Ferdinands RRK. Throwing velocity and accuracy in elite and subelite cricket players: A descriptive study. EJSS 2007;7(4):231-237. [http://dx.doi. org/10.1080/17461390701733793]

3. Bartlett RM. The science and medicine of cricket: An overview and update. J Sports Sci 2003;21:733-752. [http://dx.doi.org/10.1080/0264041031000140257]

4. MacDonald DC, Cronin JB, Stretch RA, Mills J. Wicket-keeping in cricket: A literature review. Int J Sports Sci Coach 2012;8(3). [http://dx.doi.org/10.1260/1747-9541.8.3.531]

5. Lord's. The Laws of Cricket. http://www.lords.org/data/files/laws_of cricket_2003-8685.pdf (accessed 27 May 2012).

6. Shilbury D. An analysis of fielding patterns of an 'A' grade cricket team. Sports Coach 1990:13(4):41-44

7. Scott K, Kingsbury D, Bennett S, Davids K, Langley M. Effects of cricket ball colour and illuminance levels on catching behaviour in professional cricketers. Ergonomics 2000;43(10):1681-1688. [http://dx.doi.org/10.1080/001401300750004087]

8. Wickstrom RL. Fundamental Motor Patterns. Philadelphia, USA: Lea \& Febiger, 1983.

9. Hopwood MJ, Mann DL, Farrow D, Nielsen T. Does visual-perceptual training augment the fielding performance of skilled cricketers. Int J Sports Sci Coach 2011;6(4):523-535. [http://dx.doi.org/10.1260/1747-9541.6.4.523]

10. Elliott BC, Anderson G. Age-related difference in high performance overarm throwing. J Hum Mov Stud 1990;18:1-24.

11. Cook DP, Strike SC. Throwing in cricket. J Sports Sci 2000;18(12):965-973. [http:// dx.doi.org/10.1080/793086193] 
12. Stretch RA, Goslin B. Validity and reliability of an objective test of cricket skills. Aust J Sci Med Sport 1987:18-23.

13. Bland C. Dynamic Cricket. Rhodesia: The College Press, 1969.

14. Noakes TD, Durandt JJ. Physiological requirements of cricket. J Sports Sci 2000;18:919-929. [http://dx.doi.org/10.1080\%2F026404100446739]

15. Croft JL, Button C, Dicks M. Visual strategies of sub-elite cricket batsmen in response to different ball velocities. Hum Mov Sci 2010;29:751-763. [http://dx.doi. org/10.1016/j.humov.2009.10.004]

16. Müller S, Abernathy B, Reece J, et al. An in-situ examination of the timing of information pick-up for interception by cricket batsmen of different skill levels. Psychol Sport Exerc 2009;10:644-652. [http://dx.doi.org/10.1016/j.psychsport.2009.04.002]

17. Land M, McCleod P. From eye movement to actions: How batsmen hit the ball. Nat Neurosci 2000;3:1340 -1345. [http://dx.doi.org/10.1038/81887]

18. Rudkin ST, O'Donoghue PG. Time-motion analysis of first-class cricket fielding. J Sci Med Sport 2007;11:604-607. [http://dx.doi.org/10.1016/j.jsams.2007.08.004]

19. Petersen C, Pyne D, Portus M, Dawson B. Validity and reliability of GPS units to monitor cricket-specific movement patterns. Int J Sports Physiol Perform 2009;4:381-393.
20. Petersen C, Pyne D, Portus M, Dawson B. Comparison of player movement patterns between 1-Day and Test cricket. J Strength Cond Res 2011;25(5):1368-1373.

21. Johnstone J, Ford P. Physiologic profile of professional cricketers. J Strength Cond Res 2010;24(11):2900-2907. [http://dx.doi.org/10.1519/JSC.0b013e3181bac3a7]

22. Stretch RA. Anthropometric profile and body composition changes in first-class cricketers. S Afr J Res Sport Phys Educ Recreation 1991;14(2):57-64.

23. Stretch RA. Anthropometric profile of first-class cricketers. S Afr J Res Sport Phys Educ Recreation 1987;10(1):65-75.

24. Bourdon P, Savage B, Done R. Protocols for the physiological assessment of cricket players. In: Gore CJ, ed. Physiological Tests for Elite Athletes. Champaign, USA: Human Kinetics, 2000:238-243.

25. Portus M, Kellett A, Karppinen S, Timms S. Cricket players. In: Tanner RK, Gore CJ, eds. Physiological Tests for Elite Athletes. 2nd ed. Torrens Park: Human Kinetics, 2013.

26. Fletcher JG. Calories and Cricket. Lancet 1955;1:1165-1166.

27. Petersen C, Pyne D, Portus M, Dawson B. Quantifying positional movement patterns in Twenty20 cricket. Int J Perform Anal Sport 2009;9:165-170. 